\title{
Oxygen self-diffusion along high diffusivity paths in forsterite
}

\author{
Hisayoshi Yurimoto ${ }^{1}$, Masana Morioka ${ }^{2}$ and Hiroshi NAgASAWA ${ }^{3}$ \\ Institute of Geoscience, The University of Tsukuba, Tsukuba, Ibaraki $305^{1}$ \\ Radioisotope Centre, University of Tokyo, Yayoi, Bunkyo-ku, Tokyo $113^{2}$, \\ and Department of Chemistry, Gakushuin University, \\ Mejiro, Toshima-ku, Tokyo $171^{3}$, Japan
}

(Received September 3, 1991; Accepted June 11, 1992)

\begin{abstract}
Diffusion profiles of ${ }^{18} \mathrm{O}$ tracer in single crystals of forsterite following anneals at $1100^{\circ}$ and $1200^{\circ} \mathrm{C}$ have been determined by a depth profiling technique using secondary ion mass spectrometry. The diffusion penetration profiles showed error function forms with developed tails. The profiles were analyzed in terms of diffusion in the forsterite lattice together with a contribution from dislocation. The present results of lattice diffusion lie in the range of the extrapolation of lines on Arrhenius plots reported previously for intracrystalline diffusion in forsterite. The dislocation diffusion coefficients are about $10^{4}$ times faster than the lattice diffusion at the same temperature. Diffusion along the dislocations takes place within a pipe with radius of about $0.1 \mathrm{~nm}$ surrounding the dislocation line. Based on the above results, $O$ diffusion along high diffusivity paths, such as dislocations and grain boundaries, play an important role on the diffusional creep in olivine under laboratory condition.
\end{abstract}

\section{INTRODUCTION}

Rheology of olivine is of particular importance in the dynamics of the earth's interior since olivine is the predominant phase in the upper mantle. Recently, Karato et al. (1986) found a diffusional creep regime for polycrystalline olivine in laboratory. Ozawa (1989) presented the first evidence that spinel in upper-mantle rock deformed by a diffusional creep mechanism. Karato et al. (1986) has argued that the diffusional creep mechanism may also control the rheology of olivine at low stress and small grain size which correspond to the conditions in the earth.

The self-diffusion of ionic species in minerals controls the diffusional creep, i.e., diffusional creep rate is controlled by the slowest moving ionic species diffusing over its fastest path (e.g. Gordon, 1985). Since forsterite is the $\mathrm{Mg}$ endmember of mantle olivine which contains about $10 \%$ fayalite component, analysis of diffusion in forsterite is one of the most basic key to unders- tand the physical properties of rheology in mantle. In this aspect, many studies have been made to determine lattice diffusion of $\mathrm{O}$ or $\mathrm{Mg}$ in forsterite (Sockel et al., 1980; Reddy et al., 1980; Jaoul et al., 1980; Morioka, 1981; Jaoul et al., 1981).

In the case of $\mathrm{MgO}$ and $\mathrm{Al}_{2} \mathrm{O}_{3}$, it has been shown that diffusion along high diffusivity paths plays an important role in the diffusional creep phenomena (see Gordon, 1985). For fast diffusion in olivine, Watson (1986) has made a pioneer work to determine $\mathrm{O}$ grain boundary diffusion coefficients using natural samples. However, the grain boundary diffusion is not self-diffusion but chemical diffusion because the experiments have been done under chemical activity gradients.

We have investigated diffusion mechanism in minerals using several techniques and discussed implications for geo-scientific events (Morioka, 1980, 1981, 1983; Nagasawa, 1983; Morioka et al., 1985; Yurimoto and Nagasawa, 1989; Yurimoto et al., 1989). This paper outlines results of oxygen diffusion along high diffusivity 
paths in forsterite single crystals. To our knowledge, this paper presents, for the first time, a direct measurement of $\mathrm{O}$ self diffusion rate in olivine along high diffusivity paths; although preliminary studies have been presented by Yurimoto et al. (1990 a,b). In this respect, we report a new set of diffusion data for forsterite, particularly for the purpose of demonstrating the application of the data to a high temperature creep mechanism of olivine.

\section{Sample Preparation and Diffusion AnNealing}

Forsterite single crystals were synthesized by the Czochralski (CZ) method. The starting reagents, $\mathrm{MgO}$ and $\mathrm{SiO}_{2}$, supplied by Wako Pure Chemical Co. of quality better than $3 \mathrm{~N}$, were weighed in the correct stoichiometric proportions for $\mathrm{Mg}_{2} \mathrm{SiO}_{4}$, mixed and pre-heated at $1250^{\circ} \mathrm{C}$ for several hours in air, and then loaded into an Ir crucible. A continuous gas flow of nitrogen at a rate of $200-300 \mathrm{ml} \mathrm{min}^{-1}$ was introduced during the crystal growth. Good quality forsterite crystal free from visible internal imperfections such as cracks, gas bubbles and any other inclusions were pulled along the [100] direction at a rate of $3 \mathrm{~mm}$ hour $^{-1}$ and rotation rate of $10 \mathrm{rpm}$. In this way, crystals were obtained up to $30 \mathrm{~mm}$ in diameter and $100 \mathrm{~mm}$ in length. The conditions for synthesis were similar to those employed by Morioka (1983).

The crystals were oriented by the back-reflec- tion Laue-photograph method and cut using a low-speed diamond saw into rectangular prisms with (001) surfaces of $5 \times 5 \mathrm{~mm}$ and a height of 2 $\mathrm{mm}$ along the crystallographic $c$-axis. One surface was polished to $0.1 \mu \mathrm{m}$ diamond paste. After polishing, the crystals were pre-annealed at $1200^{\circ} \mathrm{C}$ in air for 10 hours in order to keep the dislocation conditions of the crystal surface constant during diffusion annealing.

Forsterite crystals enveloped in a Pt plate (1 $\mathrm{mm}$ thickness) were annealed with focused infrared lamps in an ${ }^{18} \mathrm{O}$-enriched $\mathrm{CO}_{2}$ atmosphere $\left(99 \%{ }^{18} \mathrm{O}\right.$ enrichment and $\left.6.4 \mathrm{kPa}\right)$ at temperatures of 1100 and $1200^{\circ} \mathrm{C}$. The temperature was measured on the crystal surface by a $\mathrm{Pt}-\mathrm{Pt} \cdot \mathrm{Rh}$ thermocouple, and regulated by PID control system. The temperature fluctuations were within $\pm 3^{\circ} \mathrm{C}$ of the set temperature. Although temperature gradient between the thermocouple and the center of the sample could not be measured directly, the distance between them is only $1 \mathrm{~mm}$ and the diffusion in this study occurred only in the region near the surface. Thus the systematic error from the true anneal temperature is believed to be within $\pm 5^{\circ} \mathrm{C}$. That present results of lattice diffusion (Table 1) lie in the range of the previous reports (Sockel et al., 1980; Reddy et al., 1980; Jaoul et al., 1980; Jaoul et al., 1981) also supports the estimation of temperature systematic error. The durations of the annealing ranged from 1 hour to 4 hours.

Table 1. Oxygen self-diffusion coefficients on forsterite along c-axis

\begin{tabular}{ccccc}
\hline $\begin{array}{c}\text { Temperature } \\
{ }^{\circ} \mathrm{C}\end{array}$ & $\begin{array}{c}\text { Anneal time } \\
\text { sec. }\end{array}$ & $\mathrm{m}^{2} \mathrm{sec}^{1}$ & $\begin{array}{c}\delta D^{\mathrm{b}} \\
\mathrm{m}^{3} \mathrm{sec}^{-1}\end{array}$ & $\begin{array}{c}D^{\mathrm{d}} \\
\mathrm{m}^{2} \mathrm{sec}^{-1}\end{array}$ \\
\hline 1100 & 3960 & $3.20 \pm 1.54 \times 10^{-21}$ & $2.1 \pm 1.1 \times 10^{-28}$ & $1.7 \pm 1.3 \times 10^{-17}$ \\
1100 & 15060 & $2.81 \pm 0.51 \times 10^{-21}$ & $2.1 \pm 2.0 \times 10^{-28}$ & $3.0 \pm 2.9 \times 10^{-17}$ \\
1100 & 14400 & $2.28 \pm 0.66 \times 10^{-21}$ & $4.1 \pm 2.2 \times 10^{-28}$ & $5.6 \pm 3.7 \times 10^{-17}$ \\
1200 & 8208 & $6.67 \pm 1.26 \times 10^{-21}$ & $1.6 \pm 0.5 \times 10^{-28}$ & $2.0 \pm 0.8 \times 10^{-17}$ \\
1200 & 3654 & $1.38 \pm 0.40 \times 10^{-20}$ & $2.7 \pm 1.2 \times 10^{-27}$ & $3.9 \pm 1.9 \times 10^{-16}$ \\
1200 & 9000 & $3.97 \pm 1.50 \times 10^{-21}$ & $7.5 \pm 4.6 \times 10^{-28}$ & $9.9 \pm 7.2 \times 10^{-17}$ \\
\hline
\end{tabular}

$D^{\prime}:$ lattice diffusion coefficient, $D^{b}:$ grain boundary diffusion coefficient, $D^{d}:$ dislocation diffusion coefficent, and $\delta:$ grain boundary width.

The errors in the diffusion coefficients represent the standard deviation of several SIMS analyses.

$A$ dislocation pipe radius $a=0.1 \mathrm{~nm}$ has been used to calculate $D^{d}$. 


\section{Measurement of Concentration Gradients}

The distribution of oxygen isotopes in the annealed specimens was determined by a depthprofiling analysis with a Cameca IMS-3F ion microprobe. The basic conditions for measurement are described elsewhere in detail (Yurimoto et al., 1989).

The primary beam was mass filtered ${ }^{16} \mathrm{O}^{-}$accelerated to 5.5 or $8 \mathrm{keV}$ and adjusted for a beam current of 20 to $50 \mathrm{nA}$ and a spot size of about $50 \mu \mathrm{m}$ in diameter. The primary beam was rastered over a $350 \times 350 \mu \mathrm{m}$ area during irradiation in order to bore holes with flat bottoms into the forsterite. Secondary ions from the sputtering process were collected from the central area of $60 \mu \mathrm{m}$ in diameter using a mechanical aperture. A multibeam interferometer was used to measure the final crater depth. The sputter rate was constant as shown in Yurimoto et al. (1989).

The intensities of the negative secondary ions ${ }^{16} \mathrm{O}^{-},{ }^{17} \mathrm{O}^{-},{ }^{18} \mathrm{O}^{-}$and ${ }^{28} \mathrm{Si}^{-}$were measured. Molecular ion interferences were absent on the masses 16,18 and 28 . There was ${ }^{16} \mathrm{O}^{1} \mathrm{H}^{-}$interference for mass 17 . Since the interference was sensitive to residual gases in the sample chamber of the SIMS, the chamber vacuum was maintained below $0.5 \mu \mathrm{Pa}$ during the measurement. Moreover, in order to reduce the interference, energy filtering was achieved by offsetting the sample accelerating voltage $(+100 \sim$ $+150 \mathrm{~V}$ ) and the energy band-pass was set at $\pm 5 \mathrm{eV}$. In spite of these setting, a few amount of ${ }^{16} \mathrm{OH}$ interference was still remaining as shown in Fig. 1. The interference produced no problems to determine $\mathrm{O}$ diffusion coefficients in this study because we used ${ }^{18} \mathrm{O}$ isotope as self diffusion tracer.

The possible problems caused by electrostatic charging under ion beam bombardment were eliminated by depositing $\sim 20 \mathrm{~nm}$ of gold on the sample surface and by feedback of the shift of the maximum voltage of the ion kinetic energy distribution of mass 28 .

Since the secondary ion signal for ${ }^{16} \mathrm{O}^{-}$was diluted with the primary beam (Yurimoto et al., 1989), the intensity ratio ${ }^{18} \mathrm{O}^{-} /{ }^{28} \mathrm{Si}^{-}$was used to
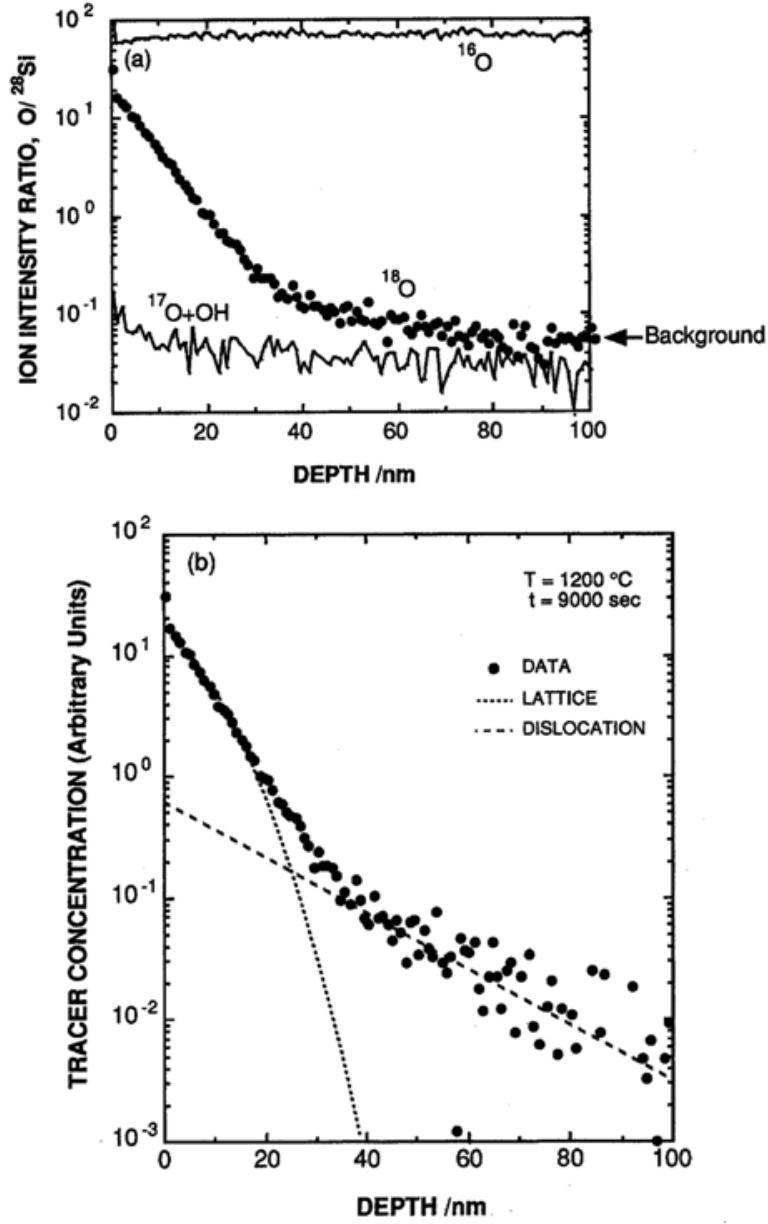

Fig. 1. (a) Diagram showing secondary ion intensity ratio against ${ }^{28} \mathrm{Si} v \mathrm{v}$. depth from surface for forsterite along the c-axis. The background level of ${ }^{18} \mathrm{O}$ which is the initial ${ }^{18} \mathrm{O}$ concentration in the forsterite is shown by arrow. (b) ${ }^{18} \mathrm{O}$ penetration profile in forsterite along the c-axis showing contributions from diffusion in the lattice, and along dislocations. The background signal corresponding to natural ${ }^{18} \mathrm{O}$ has been subtracted.

obtain the penetration plot of ${ }^{18} \mathrm{O}^{-}$in forsterite. The absence of chemical gradients near or under the crystal surface was confirmed by the signals for ${ }^{28} \mathrm{Si}^{-}$. Therefore, the intensity ratio is directly proportional to the isotopic concentration of ${ }^{18} \mathrm{O}^{-}$.

\section{Results}

For a semi-infinite medium and for a con- 


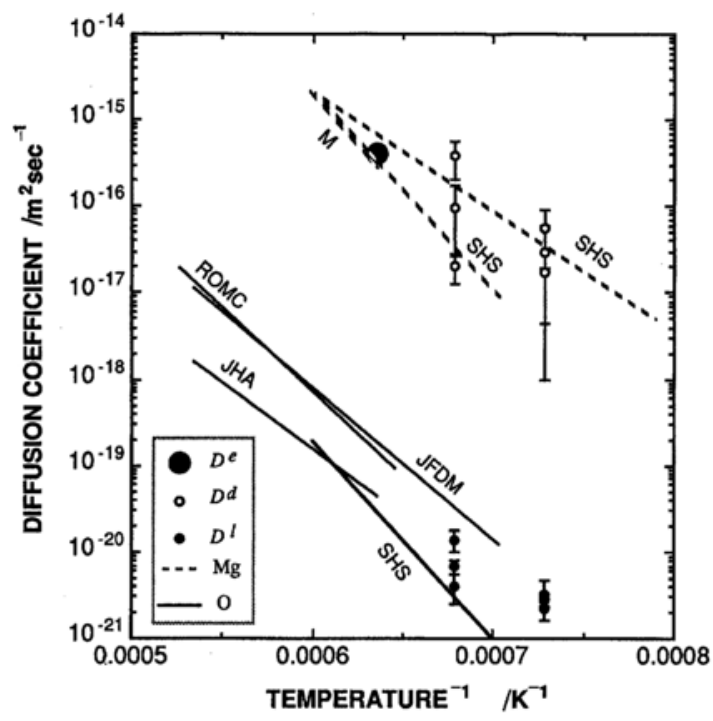

Fig. 2. Plot of the logarithm of the lattice and the dislocation diffusion coefficients for ${ }^{18} \mathrm{O}$ along the $\mathrm{c}$ axis in single crystal forsterite as a function of reciprocal temperature, with previously reported results for the self-diffusion of $O$ and $M g$. The error bars represent the standard deviation. SHS denotes Sockel et al. (1980); ROMC, Reddy et al. (1980); JFDM, Jaoul et al. (1980); M, Morioka (1981); JHA, Jaoul et al. (1983); and $D^{e}$ was determined by Karato et al. (1986).

stant concentration source with a surface concentration $C_{0}$, the lattice diffusion coefficient $D^{1}$ is written as (Crank, 1975):

$$
\frac{C-C_{0}}{C_{1}-C_{0}}=\operatorname{erf} \frac{y}{\sqrt{D^{1} t}}
$$

where $C$ is the isotopic concentration at depth $y$ from the surface, $C_{1}$ is the initial isotopic concentration in the crystal and $t$ is the annealing time. A typical penetration plot in forsterite is presented in Fig. 1. The penetration profile can be clearly resolved into an error function region, which satisfies the relation in eq. (1), plus a tail region. The error function and the tail regions can be ascribed to the result of lattice diffusion and the fast diffusion of ${ }^{18} \mathrm{O}$ along high diffusivity paths, such as dislocations at the crystal surface, respectively (Le Claire and Rabinovitch,
1984). By the same reasons as Yurimoto et al. (1989) and Sakaguchi et al. (1992) we recognized that the tail can be ascribed to the fast diffusion of ${ }^{18} \mathrm{O}$ along dislocations introduced into the surface region of the crystal by mechanical treatment. By analyzing the shapes of the tail, we determined diffusion rates of $\mathrm{O}$ along the dislocations and along the low angle tilt boundaries $^{1}$ according to the method developed by Yurimoto and Nagasawa (1989) and Le Claire (1963), respectively. The substance of the method is described in Appendix.

Table 1 summarizes the average lattice and dislocation diffusion coefficients, $D^{1}$ and $D^{\mathrm{d}}$, and the product of grain boundary width $\delta$ and grain boundary diffusion coefficient $D^{\mathrm{b}}$ along the $c$ axis for forsterite determined in the present work, along with the conditions under which these values were obtained. A plot of the logarithm of the diffusion coefficient as a function of reciprocal temperature is presented in Fig. 2.

The present results of lattice diffusion lie in the range of the extrapolation of lines reported previously (Sockel et al., 1980; Reddy et al., 1980; Jaoul et al., 1980; Jaoul et al., 1983). The dislocation diffusion coefficient is about $10^{4}$ times faster than the lattice diffusion at the same temperature. The diffusivity along dislocations is nearly equal to the self-diffusion coefficients for $\mathrm{Mg}$ in forsterite. The radius $a$ of the diffusion pipes along the $c$-axis, which correspond to the high diffusivity paths, was calculated to be $0.1 \pm 0.05 \mathrm{~nm}$.

\section{Discussion}

Recently experimental results have indicated that olivine rheology is controlled by a diffusional creep mechanism at low stresses and small grain sizes (Karato et al., 1986). In the diffusional creep regime, strain accommodation occurs by diffusion of the ionic species which constitute the rock. In general the diffusional creep 
of solids follows the following relation:

$$
\dot{\varepsilon}=A \frac{\Omega \sigma}{b^{2} R T} D^{\mathrm{e}}
$$

where $\dot{\varepsilon}$ is the strain rate, $A$ a numerical constant dependent on the geometry of flow ( $A=14$ for small strain diffusion creep) (see Raj and Ashby, 1971; Ashby et al., 1978; Spingarn and Nix, $1978), \Omega$ the molar volume, $\sigma$ the stress deviator, $b$ the grain size, $R$ the gas constant, $T$ the absolute temperature and $D^{\mathrm{e}}$ the effective diffusion coefficient. $D^{\mathrm{e}}$ is given by

$$
D^{\mathrm{e}}=1 / \Sigma\left(v_{i} / D_{i}^{\mathrm{e}}\right)
$$

(Gordon, 1975, 1985), where $v_{i}$ and $D_{i}^{\mathrm{e}}$ are the stoichiometric coefficient of the ith species in the material and its effective diffusion coefficient, respectively.

Since effective diffusion of an ion comprises the diffusion through lattice and through high diffusivity paths, effective diffusion rates change with the length of anneal time. Harrison (1961) classified the form of effective diffusion as typeA, -B and -C. Type-A diffusion is observed when $\left(D_{i}^{1} t\right)^{1 / 2} \geq 300 L$, where $D_{i}^{1}$ is $D^{1}$ of the ith species, and $L$ is the average distance between dislocations. When this condition is satisfied, $D_{i}^{\mathrm{e}}$ can be described by the relation of Hart (1957):

$$
D_{i}^{\mathrm{e}}=f D_{i}^{\mathrm{d}}+(1-f) D_{i}^{1}
$$

where $f$ is the volume fraction of the material in the dislocation, and $D_{i}^{\mathrm{d}}$ is $D^{\mathrm{d}}$ of the ith species. Type-C diffusion is observed only when the anneal time is so short that $\left(D_{i}^{1} t\right)^{1 / 2} \ll a$. In this case, diffusion from the dislocation pipe into the surrounding lattice can be neglected and $D_{i}^{\mathrm{e}}$ is characterized by $D_{i}^{d}$. When the condition for neither type-A nor type-C is satisfied, the diffusion is Type- $B$ and the diffusion coefficient is complicated because the effective diffusion both along the dislocation and into the surrounding lattice must be considered. The experimental conditions of the present study correspond to type-B diffusion.

Since components of forsterite are $\mathrm{Mg}, \mathrm{Si}$, and $\mathrm{O}, D^{\mathrm{e}}$ is determined by $D_{i}^{\mathrm{e}}$ of these elements as shown in eq. (3). Values for $D^{1}$ of $\mathrm{O}$ and $\mathrm{Mg}$ in forsterite have been measured by several works (Sockel et al., 1980; Reddy et al., 1980; Jaoul et al., 1980; Morioka, 1981; Jaoul et al., 1983). Despite the differences in sample characteristics, annealing condition and analytical method, the results are fairly consistent (cf. Fig. 2). Values for $D^{1}$ of $\mathrm{Si}$ in forsterite in published works, on the other hand, show large discrepancies (Sockel et al. 1980; Jaoul et al. 1981). Although the most recent work of Si diffusion in natural olivine confirms that $\mathrm{Si}$ is the slowest lattice diffusing species (Houlier et al., 1990), no investigations have been made for the $\mathrm{Si}$ diffusion along high-diffusivity paths. Therefore, in this paper we will mainly discuss only the effect of diffusivities of $\mathrm{O}$ and $\mathrm{Mg}$. Further work on $\mathrm{Si}$, especially for enhancement along high-diffusivity paths, is necessary for a strict quantitative test.

Since most of the creep experiments were performed within the range of several hours (e.g. Mackwell et al., 1985; Karato et al., 1986), $\left(D^{1} t\right)^{1 / 2}$ of $\mathrm{O}$ in the forsterite lattice is at most several tens of nanometers at $1300^{\circ} \mathrm{C}$, which is two orders of magnitude larger than the diffusion pipe radius determined in this study, and three orders of magnitude smaller than the grain size in the diffusional creep regime for olivine as reported by Karato et al. (1986). Moreover, since deformation of olivine continued in steady strain rate during the run duration, the effective time of diffusion for the deformation may be much shorter than the total run time. Therefore, the $D_{\mathrm{o}}^{\mathrm{e}}$ in the laboratory creep experiments is classified as Types-B to $-\mathrm{C}$, and with a value nearly equal to $D_{\text {od }}^{\text {d }}$.

The $\left(D^{1} t\right)^{1 / 2}$ for $\mathrm{Mg}$ in the forsterite lattice is several micrometers at $1300^{\circ} \mathrm{C}$, one hour, which is much greater than the typical spacing (calculated from average dislocation density reported by Karato et al., (1986)) between dislocations and is comparable to the grain radius in the diffusional creep regime. The $D_{\mathrm{Mg}}^{\mathrm{e}}$ is classified as nearly Type-A, and can be described by eq. (4). Since Karato et al., (1986) reported that the dislocation density in the diffusional creep regime is about $5 \times 10^{11} \mathrm{~m}^{-2}$, it can be con- 
cluded from eq. (4) that $D_{\mathrm{Mg}}^{\mathrm{e}}$ will be significantly influenced by diffusion along dislocations or along grain boundaries when $D^{\mathrm{d}} / D^{1}$ is greater than $2 \times 10^{8}$. To the best of the authors' knowledge, no observations have been made for diffusion coefficients of cations along high diffusivity paths in forsterite. However, since $D^{\mathrm{d}} / D^{1}$ for cation is $\sim 10^{5}$ for $\mathrm{MgO}$ (Sakaguchi $e t$ al. 1989) and $\sim 10^{4}$ for $\mathrm{NiO}$ (Atkinson and Taylor, 1979), it is unlikely that such high diffusion coefficient of $\mathrm{Mg}$ along dislocations achieved in forsterite. Therefore, $D_{\mathrm{Mg}}^{\mathrm{e}}$ would not be much enhanced by dislocations or grain boundaries under the creep experimental condition, i.e. $D_{\mathrm{Mg}}^{\mathrm{d}} \gg D_{\mathrm{Mg}}^{\mathrm{e}} \geq D_{\mathrm{Mg}}^{\mathrm{l}} \approx D_{\mathrm{O}}^{\mathrm{d}}=D_{\mathrm{O}}^{\mathrm{e}}$.

Since diffusional creep rate will be controlled by the slowest moving ionic species diffusing over its fastest path (Gordon, 1985), it is conceivable that effective diffusion of $\mathrm{O}$ controls the diffusional creep rather than that of $\mathrm{Mg}$. In fact, effective diffusion coefficient for diffusional creep for mantle olivine determined by Karato et al. (1986) lies in the range of the extrapolation for $\mathrm{O}$ dislocation diffusion in forsterite (Fig. 2).

The creep experiments in laboratories were performed at higher stress and higher strain rate than in the earth. When laboratory results are extrapolated to the lower stress levels in the earth, it is necessary to take it into consideration that

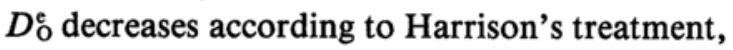
which shows that the effective diffusion coefficient is strongly dependent on grain size, sub grain size, dislocation density, dislocation distribution and strain rate. Therefore, if the diffusional creep mechanism dominates the rheology of olivine in the upper-mantle, the rheological properties must be mainly controlled by the lattice diffusion of the slowest species.

\section{Summary AND Conclusions}

Oxygen diffusion penetrations along the $c$ axis from the surface into forsterite single crystal have been analyzed by SIMS after treatment at the temperatures of $1100^{\circ} \mathrm{C}$ and $1200^{\circ} \mathrm{C}$. The present results for $\mathrm{O}$ lattice diffusion lie in the range of the extrapolation of lines reported previously.
The dislocation diffusion coefficients for $\mathrm{O}$ are about $10^{4}$ faster than the lattice diffusion coefficient at the same temperature. The $O$ diffusivity along dislocations is nearly equal to the self-diffusion coefficients for $\mathrm{Mg}$ in forsterite. The fast diffusivity region of a dislocation can be pictured as a pipe with $0.1 \mathrm{~nm}$ radius.

The diffusional creep of olivine previously observed in laboratories could be controlled by the $\mathrm{O}$ diffusion along high diffusivity paths, such as dislocations. When laboratory results are extrapolated to lower stress levels in the earth, it is necessary to correct for the fact that $D_{\mathrm{O}}^{\mathrm{e}}$ decreases according to the Harrison's treatment.

Acknowledgments-The authors are grateful to K. Kurita, S. Sueno and I. Sakaguchi for valuable discussions and assistance in SIMS analysis. The authors also thank O. Jaoul and an anonymous referee for constructive reviews of the manuscript. Partial support for this study was derived from a Grant in Aid for Scientific Research from the Ministry of Education of Japan.

\section{REFERENCES}

Ashby, M. F., Edward, G. H., Davenport, J. and Verrall, R. A. (1978) Application of bound theorems for creeping solids and their application to large strain diffusional flows. Acta Metall. 26, 1379-1388

Atkinson, A. and Taylor, R. I. (1979) The diffusion of $\mathrm{Ni}$ in the bulk and along dislocations in $\mathrm{NiO}$ single crystals. Phil. Mag. A 39, 581-595.

Atkinson, A. and Taylor, R. I. (1981) The diffusion of ${ }^{63} \mathrm{Ni}$ along grain boundaries in nickel oxide. Phil. Mag. A, 43, 979-998.

Crank, J. (1975) The Mathematics of Diffusion (2nd ed), Clarendon Press, 414p. Gerard, O. and Jaoul, O. (1989) Oxygen diffusion in San Carlos olivine. $J$. Geophys. Res. 94, 4119-4128.

Gordon, R. S. (1975) Ambipolar diffusion and its application to diffusion creep. In Mass Transport Phenomena in Ceramics, Mater. Sci. Res., vol. 9 (edited by A. R. Cooper and A. H. Heuer), pp. 445-464, Plenum, New York.

Gordon, R. S. (1985) Diffusional creep phenomena in polycrystalline oxides. In Point Defects in Minerals, Geophys. Monogr. Ser., vol. 31 (edited by R. N. Schock), pp. 132-140, AGU, Washington, D. C.

Harrison, L. G. (1961) Influence of dislocations on diffusion kinetics in solids with particular reference to the alkali halides. Trans. Faraday Soc. 57, 1191- 
1199.

Hart, E. W. (1957) On the role of dislocations in bulk diffusion. Acta Metall. 5, 597.

Houlier, B., Cheraghmakani, M. and Jaoul, O. (1990) Silicon diffusion in San Carlos olivine. Phys. Earth Planet. Inter. 62, 329-340.

Jaoul, O., Froidevaux, C., Durham, W. B. and Michaut, M. (1980) Oxygen self-diffusion in forsterite: Implications for the high-temperature creep mechanism. Earth Planet. Sci. Lett. 47, 391397.

Jaoul, O., Poumellec, M., Froidevaux, C. and Havette, A. (1981) Silicon diffusion in forsterite: A new constraint for understanding mantle deformation. In Anelasticity in the Earth, Geodyn. Ser., vol. 4 (edited by F. D. Stacey, M. S. Paterson, and A. Nicolas), pp. 95-100, AGU, Washington, D. C. Jaoul, O., Houlier, B. and Abel, F. (1983) Study of 18 $\mathrm{O}$ diffusion in magnesium orthosilicate by nuclear microanalysis. J. Geophys. Res. 88, 613-624.

Karato, S., Paterson, M. S. and FitzGerald, J. D. (1986) Rheology of synthetic olivine aggregates: Influence of grain size and water. J. Geophys. Res. 91, 8151-8176.

Le Claire, A. D. (1963) The analysis of grain boundary diffusion measurements. Brit. J. Appl. Phys. 14, 351-356.

Le Claire, A. D. and Rabinovitch, A. (1984) The mathematical analysis of diffusion in dislocations. In Diffusion in Crystalline Solids (eds. G. E. Murch and A. S. Nowick), pp. 257-318. Academic Press.

Mackwell, S. J., Kohlstedt, D. L. and Paterson, M. S. (1985) The role of water in the deformation of olivine single crystals. J. Geophys. Res. 90, 1131911333.

Morioka, M. (1980) Cation diffusion in olivine-I. Cobalt and magnesium. Geochim. Cosmochim. Acta 44, 759-762.

Morioka, M. (1981) Cation diffusion in olivine-II. Ni$\mathrm{Mg}, \mathrm{Mn}-\mathrm{Mg}, \mathrm{Mg}$ and $\mathrm{Ca}$. Geochim. Cosmochim. Acta 45, 1573-1580.

Morioka, M. (1983) Cation diffusion in olivine-III. $\mathrm{Mn}_{2} \mathrm{SiO}_{4}$ system. Geochim. Cosmochim. Acta 47 , 2275-2279.

Morioka, M., Nagasawa, H. and Suzuki, H. (1985) Trace element diffusion in olivine: mechanism and a possible implication to natural silicate systems. In Point Defects in Minerals, Geophys. Monogr. Ser., vol. 31 (edited by R. N. Schock), pp. 116-121, AGU, Washington, D. C.

Nagasawa, H. (1983) Diffusion coefficient of O in melilite: Implication to the thermal history of Allende CAI. Lunar Planet. Sci. XIV, 538-539.

Ozawa, K. (1989) Stress-induced Al-Cr zoning of spinel in deformed peridotites. Nature 338, 141-
144.

Raj, R. and Ashby, M. F. (1971) On grain boundary sliding and diffusional creep. Trans. Metall. Soc. AIME 2, 1113-1127.

Reddy, K. P. R., Oh, S. M., Major, L. D. and Cooper, A. R. (1980) Oxygen diffusion in forsterite, J. Geophys. Res. 85, 322-326.

Ryerson, F. J., Durham, W. B., Cherniak, D. J. and Lanford, W. A. (1989) Oxygen diffusion in olivine: effect of oxygen fugacity and implications for creep. J. Geophys. Res. 94, 4105-4118.

Sakaguchi, I., Yurimoto, H. and Sueno, S. (1989) Trace element diffusion in MgO, III. Ann. Meet. Abs. Min. Soc. Jpn., 1989, 99.

Sakaguchi, I., Yurimoto, H. and Sueno, S. (1992) Calcium diffusion along high-diffusivity paths in single-crystal MgO. J. Am. Ceram. Soc, 75, 712715.

Sockel, H. G., Hallwig, D. and Schachtner, R. (1980) Investigations of slow exchange processes at metal and oxide surfaces and interfaces using secondary ion mass spectrometry. Mater. Sci. Eng. 42, 59-64.

Spingarn, J. R. and Nix, W. D. (1978) Diffusional creep and diffusionally accommodated rearrangement. Acta Metall. 26, 1389-1398.

Turnbull, D. and Hoffman, R. E. (1954) The effect of relative crystal and boundary orientations on grain boundary diffusion rate. Acta Metall. 2, 419-426.

Watson, E. B. (1986) An experimental study of oxygen transport in dry rocks and related kinetic phenomena. J. Geophys. Res. 91, 14117-14131.

Yurimoto, H. and Nagasawa, H. (1989) The analysis of dislocation pipe radius for diffusion. Mineral. $J$. 14, 171-178.

Yurimoto, H., Morioka, M. and Nagasawa, H. (1989) Diffusion in single-crystals of melilite: I. Oxygen. Geochim. Cosmochim. Acta 53, 2387-2394.

Yurimoto, H., Morioka, M. and Nagasawa, H. (1990a) Oxygen dislocation diffusion in forsterite and implications for creep. Eos 71, 626.

Yurimoto, H., Morioka, M. and Nagasawa, H. (1990b) Oxygen diffusion along high diffusivity paths in forsterite and implications for creep mechanism. Eos 71, 904.

\section{APPENDIX}

The analysis of dislocation pipe radius for diffusion and mathematical procedure for dislocation diffusion coefficient have been fully described by Yurimoto and Nagasawa (1989). We describe the substance of the procedure below.

The analysis of the high-diffusivity paths was 
generally based on the grain-boundary model and the dislocation pipe model. Le Claire (1963) has developed the equation of the grain boundary contribution $\left(\delta D^{b}\right)$ as

$\delta D^{\mathrm{b}}=0.66\left(\frac{d \ln \langle c\rangle}{\mathrm{d} y^{6 / 5}}\right)^{-5 / 3}\left(\frac{4 D^{\mathrm{l}}}{t}\right)^{1 / 2}$

where $\langle c\rangle$ is the total average concentration at depth $y$. Le Claire and Rabinovitch (1984) indicate that the dislocation array contribution appears as

$$
a^{2} D^{\mathrm{d}}=\left[\frac{A(\alpha)}{\left(\frac{\partial \ln \langle c\rangle}{\partial y}\right)}\right]^{2} D^{1}
$$

where $\mathrm{A}(\alpha)$ is an $\alpha$-dependent factor describing tail slopes, $\alpha=a /\left(D^{1} t\right)^{1 / 2}$, and the parameter of $a$ is dislocation pipe radius. Low-angle grain boundaries can be described geometrically as planer arrays of individual dislocations. According to Turnbull and Hoffman (1954), the diffusion in the direction of the dislocation pipes is written as

$$
\delta D^{\mathrm{b}}=D^{\mathrm{d}} \frac{\pi a^{2}}{L}
$$

where $L$ is the space of dislocation pipes. The grain size $(b)$, which means the space between grain boundaries, appears as

$$
b=\frac{2\left(D^{1} t\right)^{1 / 2}\left(4.1 D^{1}\left(D^{1} t\right)^{1 / 2} / \delta D^{\mathrm{b}}+1.56\right)}{\langle c\rangle_{\mathrm{Int}}^{\mathrm{b}} / c_{0}}
$$

where $\langle c\rangle_{\text {Int }}^{b}$ is the intersection value of an extrapolation of the tail back to the surface $(y=0)$ and the ordinate, and $c_{0}$ is the surface concentration. The dislocation pipe radius appears as

$$
a=\left(\frac{b L \frac{\langle c\rangle_{\text {lnt }}^{\mathrm{d}}}{c_{0}}}{2 \pi\left(D^{1} t\right)^{0.92}}\right)^{6.25}
$$

where $\langle c\rangle_{\text {Int }}^{d}$, is the intersection value of an extrapolation of the tail back to the surface $(y=0)$ and the ordinate. Therefore, $D^{\mathrm{d}}$ and a are obtained using an iteration procedure with the help of eqs. (6), (7) and (9). 